\title{
Dopamine Content and Metabolism in Mesencephalic and Diencephalic Cell Cultures: Sex Differences and Effects of Sex Steroids
}

\author{
Cordian Beyer, Christoph Pilgrim, and Ingrid Reisert \\ Abteilung Anatomie und Zellbiologie der Universität Ulm, 7900 Ulm, Germany
}

\begin{abstract}
Sexual differentiation of dopaminergic neurons was studied in gender-specific cultures. Dissociated cell cultures were prepared from di- or mesencephalon of gestational day 14 rat embryos and raised in the absence or presence of $17 \beta$ estradiol or testosterone for up to 13 days in vitro (DIV). Developmental profiles of levels of dopamine (DA) and metabolites as well as capacity for vesicular storage of the transmitter were determined by HPLC. Tyrosine hydroxylase-immunoreactive (TH-IR) neurons were counted.

Higher levels of DA were measured in female than in male cultures of both brain regions. In mesencephalic cultures, the differences in DA levels were fully accounted for by sex differences in numbers of TH-IR cells, whereas no sex differences in cell numbers were found in diencephalic cultures. Dihydroxyphenylacetic acid (DOPAC) levels and vesicular storage capacity matured faster in mesencephalic than in diencephalic cultures, but no sex differences were observed. Homovanillic acid (HVA) could not be detected except in 13-DIV mesencephalic cultures. Hormonal treatment did not erase sexual differentiation of dopaminergic neurons. Irrespective of the gender, however, both steroids decreased DA and DOPAC contents in diencephalic cultures but not in mesencephalic cultures.

It is proposed that sexual differentiation of dopaminergic systems proceeds in a region-specific fashion and that neurogenesis and development of various parameters of dopaminergic activity may be differentially affected. Sexual differentiation of dopaminergic neurons may be initiated independently of the action of gonadal steroid hormones and may subsequently be modified by differences in hormonal environment.
\end{abstract}

Several pieces of evidence suggest that central dopaminergic systems are involved in the sexual differentiation of the brain. Sex differences in dopamine (DA) metabolism begin to appear during the perinatal "critical" period of brain development (Wilson and Agrawal, 1979; Vaccari, 1980; Stanley and Watts, 1985), which, in the rat, extends from gestational day (GD) 18 to the end of the first postnatal week (MacLusky and Naftolin,

Received Apr. 9, 1990; revised Nov. 27, 1990; accepted Dec. 7, 1990.

This work was supported by Deutsche Forschungsgemeinschaft Grant $\operatorname{Re} 413$ / 2-2,3. We wish to thank Ms. Brigitte Epp and Mr. Wolfgang Podschuweit for excellent technical assistance.

Correspondence should be addressed to Professor Dr. Ingrid Reisert, Abteilung Anatomie und Zellbiologie, Universität Ulm, Postfach 4066, D-7900 Ulm, Germany.

Copyright (C) 1991 Society for Neuroscience $0270-6474 / 91 / 111325-09 \$ 03.00 / 0$
1981). Manipulation of perinatal hormonal environment results in permanent changes of transmitter turnover in tuberoinfundibular dopaminergic neurons (Demarest et al., 1981) and in changes of striatal and limbic dopamine content (Leret et al., 1987). Simerly et al. (1985a,b) described a sexual dimorphism in the distribution of dopaminergic nerve cells and fibers in the preoptic region of the rat that can be sex reversed during the critical period. Administration of nicotine, a potent excitatory drug for nigrostriatal dopaminergic neurons, to pregnant rats affects DA metabolism and receptors of pups in a sex-specific manner (Lichtensteiger et al., 1988; Fung and Lau, 1989) and abolishes the sexual dimorphism in saccharin preference in the adult offspring (Lichtensteiger and Schlumpf, 1984).

The above observations support the current concept according to which sexual differentiation of the rat brain is brought about by perinatal exposure to estrogen converted from gonadal testosterone (MacLusky and Naftolin, 1981). However, there are a few observations that do not appear to be accounted for by this concept. Gorski and Jacobson (1982) detected a sex difference in the number of radiolabeled preoptic neurons of 30-d-old rats that had been exposed to ${ }^{3} \mathrm{H}$-thymidine at GD 14. Notwithstanding later sex-specific modifications of neuron numbers by migration and cell death, the authors interpreted this observation as the result of an increased rate of early neurogenesis in females. If this is correct, the sex difference cannot be explained at present by differences in hormonal environment because the surge of testosterone that is believed to initiate development of a male brain (McEwen, 1981) does not occur until GD 17/18 (Weisz and Ward, 1980). Evidence provided by in vitro studies also indicates that sexual differentiation may be generated by mechanisms other than epigenetic action of steroids. Dopaminergic neurons growing in dissociated cell cultures prepared from di- and mesencephalon of GD-14 male or female rat embryos are characterized by differences in their capacity to take up exogenous radiolabeled DA (Engele et al., 1989b; Reisert et al., 1989). This sexual dimorphism in DA uptake was present in cultures of tissue removed before the perinatal rise of testosterone occurs in males (Weisz and Ward, 1980) and in the absence of hormonal additives to the culture medium. The objective of the present investigation was to supplement these results with determinations of other parameters indicative of dopaminergic activity, specifically, levels of endogenous dopamine and its main metabolites, as well as the capacity for vesicular storage of the transmitter.

\section{Materials and Methods}

Materials. Timed pregnant Sprague-Dawley rats were obtained from Charles River Wiga, Sulzfeld, Germany. The materials used include 
Table 1. Numbers of TH-IR cells per square centimeter in mesencephalic and diencephalic genderspecific dissociated cell cultures of GD 14

\begin{tabular}{rlllll} 
& \multicolumn{2}{l}{ Mesencephalon } & & Diencephalon & \\
\cline { 2 - 3 } \cline { 5 - 6 } & Male & Female & & Male & Female \\
\hline 3 DIV & $2927 \pm 134^{*}$ & $3344 \pm 65$ & & $1687 \pm 61$ & $1732 \pm 113$ \\
6 DIV & $2270 \pm 200^{* *}$ & $2800 \pm 109$ & & $750 \pm 58$ & $789 \pm 75$ \\
8 DIV & $2030 \pm 173^{* * *}$ & $2568 \pm 109$ & & $713 \pm 64$ & $715 \pm 49$ \\
13 DIV & $1957 \pm 156^{* * *}$ & $2447 \pm 182$ & & $700 \pm 78$ & $692 \pm 53$
\end{tabular}

Values represent means \pm SD from four independent experiments. ${ }^{*}, p \leq 0.01 ;{ }^{* *}, p \leq 0.002 ;{ }^{* * *}, p \leq 0.005 ;$ male versus female.

castrated horse serum from Behring (Marburg, Germany); minimum essential medium (MEM)-Earle's medium (powder) from Biochrom (Berlin, Germany); $3.5-\mathrm{cm}^{2}$ culture dishes from Costar (Tecnomara, Fernwald, Germany); poly-L-lysine (MW, 80,000-220,000), di- $n$-butylamine, 1-octansulfonic acid (OSA), 3,4-dihydroxyphenylacetic acid (DOPAC), 4-hydroxy-3-methoxyphenylacetic acid (HVA), 3,4-dihydroxybenzylamine (DHBA), and dopamine (DA) from Sigma (Deisenhofen, Germany); trypsin and Versene (1:5000) from Gibco/British Research Labs (Eggenstein, Germany); citric acid, sodium acetate, EDTA, and perchloric acid (PCA) from Merck (Darmstadt, Germany); methanol (HPLC grade) from Rathburn Chemicals Ltd. (Walkersburn, Scotland); reserpine from Serva (Heidelberg, Germany); monoclonal anti-rat tyrosine hydroxylase (TH) IgG (mouse) from Boehringer (Mannheim, Germany); biotinylated anti-mouse IgG (horse), fluorescein isothiocyanate (FITC)-labeled avidin-D and ABC Kit from Vector (Camon, Wiesbaden, Germany); and 3,3'-diaminobenzidine tetrahydrochloride dihydrate (DAB) from Aldrich (Steinheim, Germany). All other chemicals used were of the purest grade commercially available.

Cells and culture conditions. The preparation of dissociated cell cultures of mes- and diencephalon from fetal rat brains has been described in detail (Engele et al., 1989b; Reisert et al., 1989). Male and female fetuses were separated by inspection of the gonadal anlage. In GD 14 males, the testicular artery can be seen running along the edge of the gonad, and sex cords can be visualized by dark-field illumination. These features were absent in female fetuses. Briefly, the brains were removed on gestational day (GD) 14 (day of insemination, GD 0; crown-rump length, $10.5-11.0 \mathrm{~mm}$ ) under sterile conditions followed by enzymatic and mechanical dissociation of di- and mesencephalic tissue. Cells were plated at a density of $2.5 \times 10^{5}$ cells $/ \mathrm{cm}^{2}$ on poly-L-lysine $(1 \mu \mathrm{g} / \mathrm{ml})$ coated plastic culture dishes. Cultures were raised with phenol red-free MEM supplemented with $10 \%$ castrated horse serum, in which sex steroids were not detectable by radioimmunoassay (RIA), at $37^{\circ} \mathrm{C}$ in a water-saturated atmosphere of $5 \% \mathrm{CO}_{2}$ and $95 \%$ air. $17 \beta$-Estradiol or testosterone were added to the medium daily in final concentrations of $10^{-12}$ or $10^{-9} \mathrm{M}$, respectively. These are physiological concentrations that have been reported to occur in the fetal rat (Pasqualini and Kincl, 1985). Phenol red was avoided because it mimics estrogen effects (Berthois et al., 1986). In some experiments, reserpine $(1 \mu \mathrm{M})$ was added to the culture medium $3 \mathrm{hr}$ prior to harvesting cultured cells.

Immunocytochemistry. Catecholaminergic neurons in di- and mesencephalic cultures were identified by means of anti-tyrosine hydroxylase $(\mathrm{TH})$ immunocytochemistry. Cells immunoreactive for $\mathrm{TH}$ that occur in these cultures have been characterized as dopaminergic (Engele et al., 1989a). After rinsing the cultures with serum-free MEM and fixing in cold paraformaldehyde (4\%) for $30 \mathrm{~min}$, cells were permeabilized with $0.05 \%$ saponin in Hank's balanced salt solution for $30 \mathrm{~min}$. Cultures were incubated overnight with anti-TH at $4^{\circ} \mathrm{C}$. Anti-TH was diluted 1:750 in phosphate $(10 \mathrm{~mm})$-buffered saline containing $0.5 \%$ bovine serum albumin and $0.05 \% \mathrm{NaN}_{3}$. Antigen-antibody complex was visualized by using biotinylated anti-mouse IgG (1:500) raised in horse as a secondary antibody and FITC-labeled avidin-D for $2 \mathrm{hr}$ each (for details, see Beyer et al., 1990). Immunofluorescent preparations were screened with a Neofluar objective using a Zeiss Universal microscope (epillumination) equipped with an FITC optic and photographed with T-Max film (Kodak) rated at 400 ASA. For quantitation of TH-immunoreactive (TH-IR) cells, antigen-antibody complex was visualized by the avidin-biotin (ABC) method. Instead of FITC-labeled avidin-D, cultures were incubated for $2 \mathrm{hr}$ with biotinylated horseradish peroxidase complex. Staining was developed with DAB in the presence of
$0.01 \% \mathrm{H}_{2} \mathrm{O}_{2}$ dissolved in Tris- $\mathrm{HCl}$ buffer ( $50 \mathrm{~mm}$; $\left.\mathrm{pH}, 7.2\right)$. Numbers of immunostained perikarya were counted by screening entire cultures with a $25 \times$ objective. Parallel cultures from the same experiment were used for quantitation of TH-IR cells and HPLC analysis (see below).

Tissue collection. After $3,6,8,10$, and $13 \mathrm{~d}$ in vitro (DIV), cultivation was stopped by washing cultures twice with $\mathrm{Ca}^{2+}$-free phosphate $(10$ mM)-buffered saline ( $\mathrm{pH}, 7.2)$ at room temperature, and cells of each well were harvested with $150 \mu \mathrm{l}$ cold PCA $(50 \mathrm{~mm})$. Cells were scraped off each culture dish separately, sonicated for $10 \mathrm{sec}$, and centrifuged at $14,000 \times g$ for $5 \mathrm{~min}$ at $4^{\circ} \mathrm{C}$. Supernatants were stored at $-20^{\circ} \mathrm{C}$ until catecholamine assays were performed. Protein content of the pellets was measured according to the micromethod described by Smith et al. (1985).

HPLC analysis. Catecholamines and metabolites were separated by high-pressure liquid chromatography (model 300 B, Gynkotek, Germering, Germany) on a reversed-phase $\mathrm{C}_{18}$ column $\left(4.6 \times 125 \mathrm{~cm}^{2}\right.$; ODShypersil, $5 \mu \mathrm{m}$; Gynkotek, Germany). Flow rate was adjusted to $0.8 \mathrm{ml} /$ min, injection volume was $20 \mu \mathrm{l}$, and background pressure was approximately $1600 \mathrm{psi}$. The mobile phase consisted of a citrate-sodium acetate buffer ( $50 \mathrm{~mm}$ sodium acetate and $20 \mathrm{~mm}$ citric acid monohydrate; $\mathrm{pH}, 3.5$ ) containing $2.7 \mathrm{~mm}$ OSA, $100 \mu \mathrm{M}$ EDTA, $130 \mathrm{mg}$ di- $n$ butylamine, and $3 \%$ methanol. The mobile phase was degassed with helium. An electrochemical detector (model M 20, Gynkotek) was used to determine and quantify catecholamines. The glassy carbon working electrode was set to a potential of $+700 \mathrm{mV}$ relative to an $\mathrm{Ag} / \mathrm{AgCl}$ reference electrode. Off-set current was in the range of $0.8-1.0 \mathrm{nA}$. Fresh working standards of DA, DOPAC, and HVA were made up daily in PCA (50 mM). Standard curves were linear over a range of $0.5-200$ pmol per $20 \mu \mathrm{l}$ injection. Peaks of DA and metabolites were identified by comparison with the retention times of standards, which were 8,12 , and $18 \mathrm{~min}$ for DOPAC, HVA, and DA, respectively. The concentrations of each compound were determined by comparing peak heights with those obtained for a standard mixture. Recovery of DA and metabolites was analyzed by the peak-height-ratio method using DHBA ( $2 \mathrm{pmol})$ as an internal standard to correct for sample loss. Typical recoveries were between $79 \%$ and $86 \%$. Detection limits for DOPAC, HVA, and DA determined at a signal-to-noise ratio of $3: 1$ were 0.08 , 0.08 , and $0.1 \mathrm{pmol}$, respectively.

Statistical analysis. A total of seven independent experiments (platings) were performed. Fetuses from two to three pregnant rats were pooled and used for one plating. Four independent experiments were carried out for quantitation of DA and metabolite levels, as well as numbers of TH-IR cells. Per experiment, three to five cultures each served to determine effects of gender, cultivation time, and hormones. Material from three experiments was used for studying vesicular storage (reserpine treatment, four cultures each per gender and time point). Means \pm SD were calculated from averaged values of the four experiments. Statistical differences between means were evaluated by the $t$ test, with $p \leq 0.01$ as the criterion for statistical significance.

\section{Results}

Visualization of TH-IR neurons revealed the same marked differences in morphology between of di- and mesencephalic culture as have been described by Engele et al. (1989a). Mesencephalic TH-IR neurons were larger and had longer, more arborized processes than diencephalic TH-IR neurons (Fig. 1a,b). These regional differences persisted throughout cultivation. In 

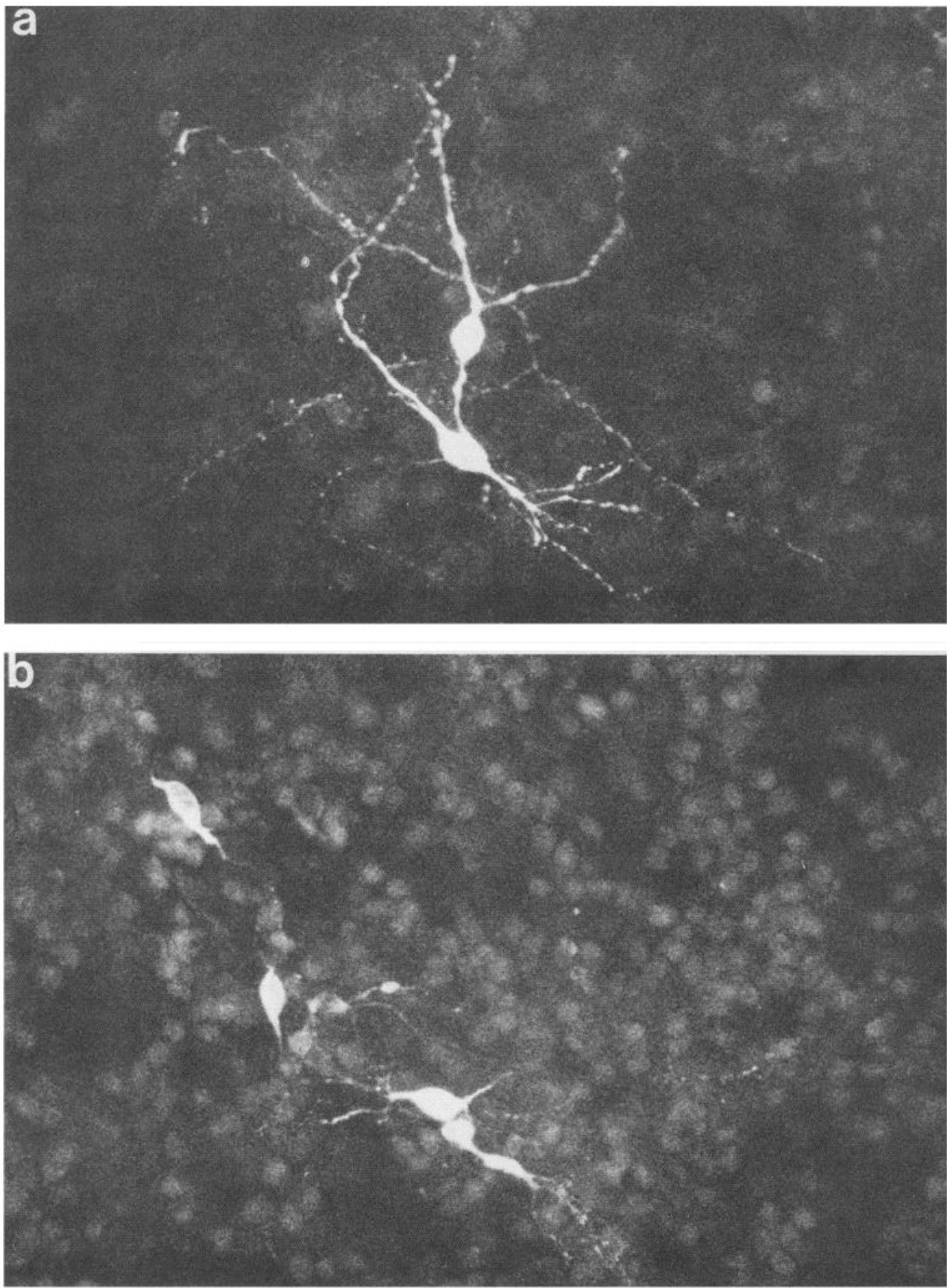

Figure 1. TH-IR cells in female mesencephalic $(a)$ and diencephalic $(b)$ cultures from GD 14 after 6 DIV visualized by indirect immunofluorescence. Note the regional differences in lengths of processes. Magnification, $400 \times$. addition, there was a transient sex difference in diencephalic cultures. As described by Reisert et al. (1989), outgrowth of neurites was faster from female than from male neurons. Table 1 gives numbers of TH-IR neurons in gender-specific cultures of di- and mesencephalon. In both regions and both genders, these numbers decreased with cultivation time. Female mesencephalic cultures were found to contain higher numbers of TH-IR cells than did male cultures. No statistically significant differences between genders were observed with diencephalic cultures. Estrogen or testosterone treatment had no significant effect on numbers of TH-IR cells (data not shown).

A representative chromatogram from a female mesencephalic culture at 13 DIV is shown in Figure 2. Measurable amounts of DA and DOPAC were present as early as 3 DIV. In mes- encephalic cultures, DA increased steeply to values of more than $1.0 \mathrm{ng}$ per well at $10 \mathrm{DIV}$ and remained on that level until DIV 13 (Fig. 3A). DOPAC contents paralleled the increase of DA until DIV 6 but stayed constant thereafter. HVA was detectable in 13-DIV cultures only ( $0.1 \mathrm{ng} /$ well; not shown). Cultures of female embryos contained significantly more DA than those from male embryos. This sex difference became apparent at DIV 6 and continued to be present at all further time points examined. No such differences were observed concerning DOPAC and HVA levels.

The developmental profile of endogenous DA and DOPAC in diencephalic cultures is shown in Figure $3 B$. DA increased only slightly during cultivation from $0.12 \mathrm{ng} /$ well at DIV 3 to $0.26 \mathrm{ng} /$ well at DIV 13. DOPAC increased dramatically during 


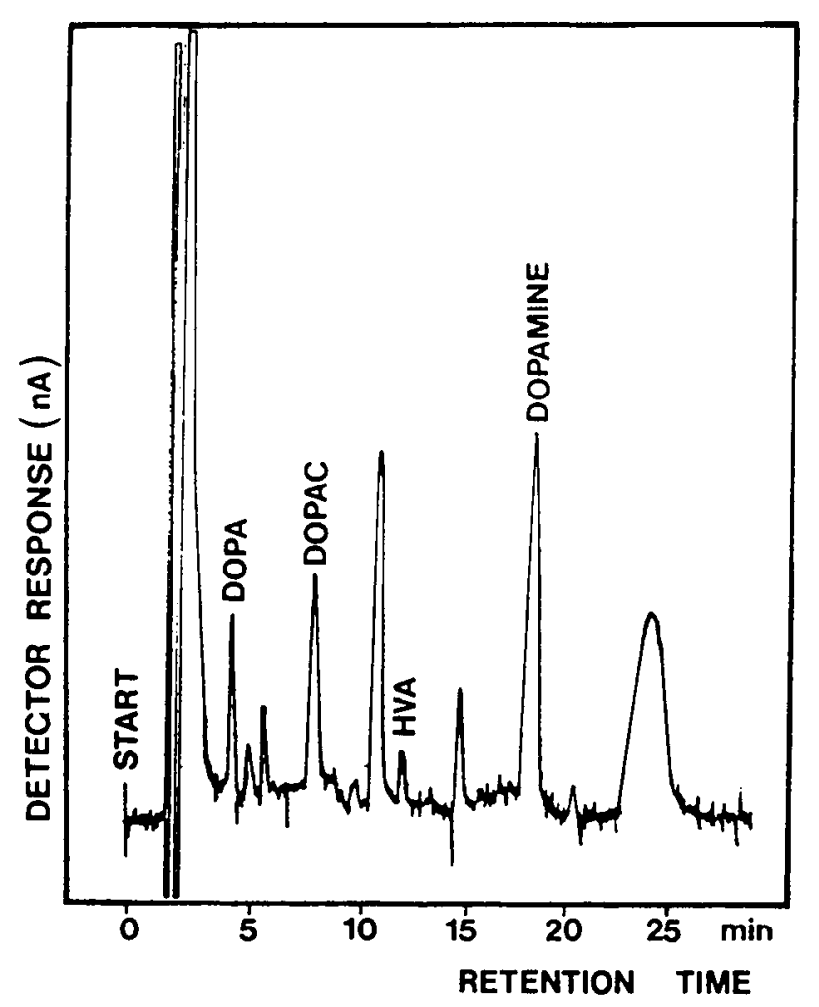

Figure 2. Chromatogram of a PCA $(50 \mathrm{~mm})$ extract from a female mesencephalic culture at 13 DIV.

the first week in culture, with highest values at DIV 8, and fell off thereafter. HVA could not be detected during the entire cultivation period. DA contents again showed sex-related differences, which became statistically significant after 10 DIV.

The capability of the cells for vesicular storage of the transmitter was investigated using reserpine treatment. Because no sex differences in reserpine sensitivity were observed, only data obtained from male tissue are given in Figure 4. In mesencephalic cultures (Fig. $4 A$ ), a reserpine effect started to appear at DIV 6. After $8 \mathrm{DIV}$, reserpine reduced vesicular DA to about $10 \%$ of that of control cultures. At the same time, endogenous DOPAC levels were elevated by about $50 \%$ compared to controls. In diencephalic cultures (Fig. $4 B$ ), a reserpine effect was first seen after 8 DIV. The maximal reduction in DA levels obtained within the experimental period was to $30 \%$ of that of controls. Elevated DOPAC levels were found at 13 DIV only. In both brain regions, reserpine treatment had no appreciable effect on HVA levels.

Figures 5 and 6 summarize the results of testosterone and $17 \beta$-estradiol treatment on transmitter and metabolite contents. Chronic administration of either steroid hormone caused a slight but significant reduction of DA at 10 and 13 DIV and of DOPAC at 13 DIV in both male and female diencephalic cultures (Fig. $5 A, B$ ). Levels of HVA were not affected by steroid hormone treatment (data not shown). Gonadal steroids did not significantly alter DA, DOPAC, or HVA levels in mesencephalic cultures of both genders (Fig. $6 A, B$ ).

\section{Discussion}

The main results emerging from the present study are sexual dimorphisms in numbers and function of dopaminergic neurons grown in a medium devoid of detectable amounts of sex steroids.
TH-IR cells, which have previously been proved to represent dopaminergic neurons (Engele et al., 1989a), were more numerous in cultures prepared from female mesencephalon than in those from male mesencephalon. Functional sex differences have been observed in cultures from both regions: Female cultures contained more endogenous DA than did male cultures. Furthermore, it is important to note that the sex differences were present in cultures of tissue that had been removed at GD 14.0 , that is, before the time when sex differences in sex hormone levels are known to develop. This is clearly in conflict with current belief, according to which sexual dimorphisms of the brain are entirely mediated by epigenetic action of gonadal hormones (MacLusky and Naftolin, 1981; Arnold and Gorski, 1984). The decisive event is thought to be the surge of testosterone in the male rat fetus at GD $17 / 18$ (Weisz and Ward, 1980), which coincides with the beginning of the critical period of sexual differentiation of the rat brain (MacLusky and Naftolin, 1981). Our findings would thus appear to support the conclusion that gender-linked differentiation of dopaminergic neurons is initiated independently of the presence of gonadal hormones and may instead be under primary genetic control.

Several arguments and as yet unsolved methodological problems caution against a hasty acceptance of such a far-reaching conclusion. For instance, steroid treatment reduced the DA content of our diencephalic cultures. Given the situation in vivo of male brains presumably being exposed to higher sex steroid levels than female brains, this is exactly what one would expect if sex steroids were responsible for the lower DA content of male dopaminergic cells. Also, the differences in gonad morphology that are present at GD 14.0, and which we have used to distinguish male from female embryos, appear to suggest the presence of functional differences, as well. Male and female gonads may already differ in production of steroids or other compounds with unknown developmental effects at this early stage. As to the production of testosterone, it should be noted that, in the experiments of Weisz and Ward (1980), the same strain of rats was used, and the age of the embryos was determined in the same way as in our investigations. However, it cannot be excluded that the RIA available at that time was not sensitive enough to detect subtle differences in hormone levels between male and female rat embryos prior to GD 18. According to the in vitro studies of Picon (1976) and Feldman and Bloch (1978), the male rat gonad begins to secrete testosterone shortly after our harvest date. It remains to be seen when the quantities of testosterone produced by the testis are large enough in comparison to androgens produced by other tissues (placenta, adrenal) to generate sex-specific differences in androgen levels. Given the possibility of strain and stock differences in developmental schedules, such hormone tests need to be performed in the very rat stock used as a tissue source for our cultures. Notwithstanding the need for supplementary information, it does not seem premature to conclude that, in some neural systems, sex differences may be determined considerably earlier than realized to date.

In the course of the present experiments, levels of DA have been determined as amounts per culture dish and were not related to protein content, because the protein content of the cultures is largely determined by glial cells. These continue to proliferate in vitro, which results in an increasing proportion of glial protein (Beyer et al., 1990). As an alternative, DA may be related to the numbers of TH-IR neurons. This calculation leads to a content of about $1.1 \mathrm{fmol}$ DA per neuron for both male 

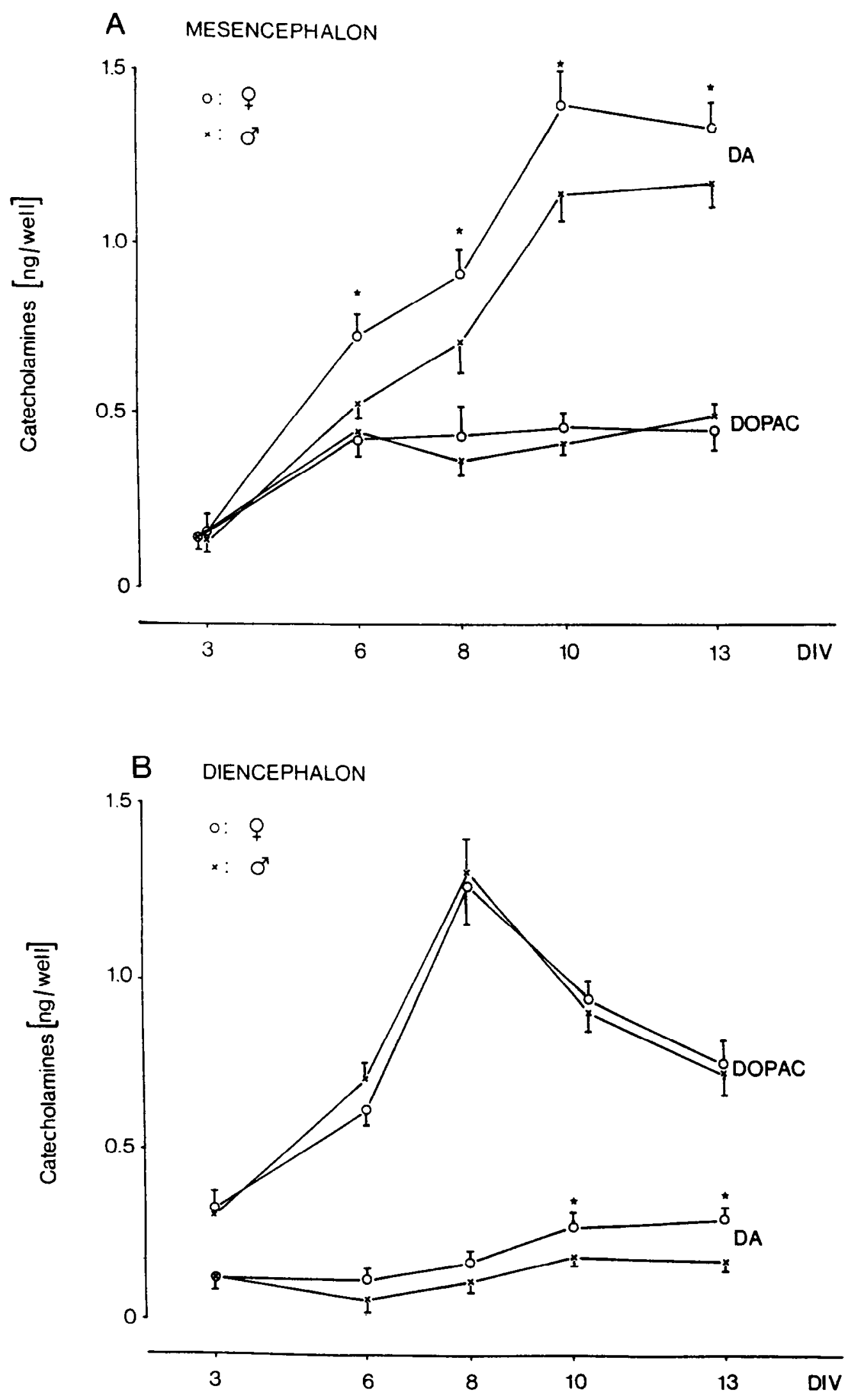

Figure 3. Developmental profile of intracellular DA and DOPAC levels in gender-specific cultures of embryonic rat mesencephalon $(A)$ and diencephalon $(B)$. Circles and crosses represent means \pm SD from four independent experiments. Asterisks, $p \leq 0.001$ for $A$, $p \leq 0.005$ for $B$, male versus female.

and female mesencephalic cultures and of 0.6 and $0.8 \mathrm{fmol} \mathrm{DA}$ per neuron for male and female diencephalic cultures, respectively, after 13 DIV. In the mesencephalon, the sex difference in DA levels can be explained on the basis of a sex difference in dopaminergic cell numbers, whereas cell numbers did not differ significantly between male and female diencephalic cultures. The sex differences in mesencephalic dopaminergic cell numbers could be the consequence either of differences in pro- 

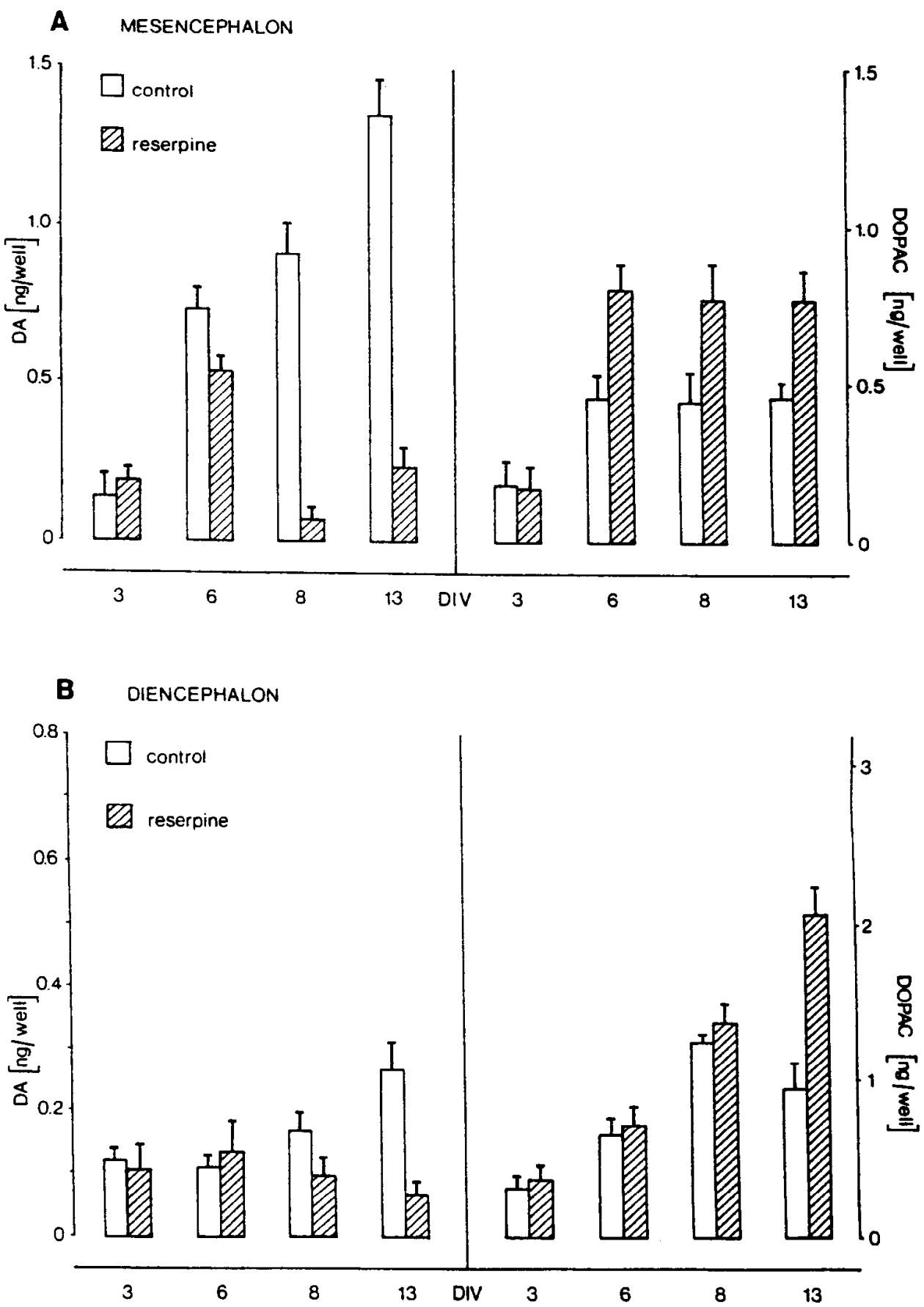

Figure 4. Effect of reserpine treatment on intracellular DA and DOPAC concentrations in male mesencephalic $(A)$ and diencephalic $(B)$ cultures. Bars represent means $\pm \mathrm{SD}$ from three independent experiments.

liferation and neurogenesis taking place before the tissue was removed or of differences in developmental schedule and/or survival of postmitotic neurons. Transient sex differences in numbers of TH-IR neurons, as demonstrated here in vitro, have also been observed in vivo during prenatal development of the rat mesencephalon (Reisert et al., 1990).

There were no sex differences in DOPAC levels in either region. This may be due to the fact that DOPAC rapidly diffuses out of the cell (Moore, 1987), which tends to obscure small differences in DOPAC production between male and female neurons, should they be present. Absence of sex differences in
DOPAC levels of the developing rat brain has also been reported in vivo (Ribary et al., 1986). Similarly, the development of vesicular storage in our cultures as probed with reserpine (Gracfc and Bönisch, 1989) did not reveal differences between genders. There were, however, considerable regional differences: In mesencephalic cultures, the capacity for vesicular storage of DA developed between 6 and 8 DIV, whereas in diencephalic cultures, this index of maturation appeared between 8 and 13 DIV. As expected, DOPAC levels rose in response to reserpine treatment only when vesicular storage of the transmitter had developed. The retardation of the onset of vesicular storage in 

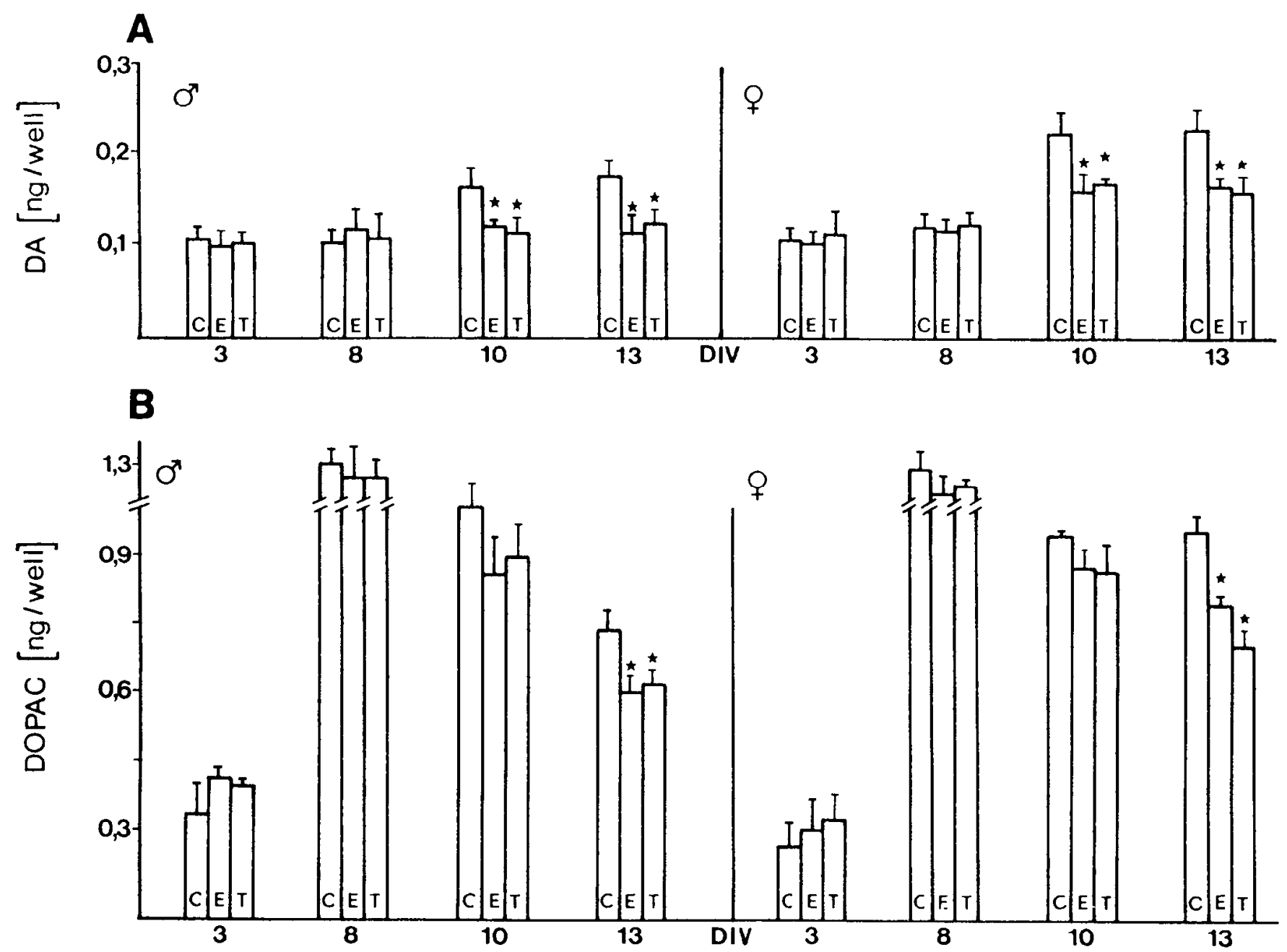

Figure 5. Effects of chronic administration of testosterone $(T)$ or $17 \beta$-estradiol $(E)$ on DA $(A)$ and DOPAC $(B)$ contents of diencephalic cultures. Bars represent means $\pm \mathrm{SD}$ from four experiments. Asterisks, $p \leq 0.005, T$ or $E$ versus controls $(C)$.

diencephalic dopaminergic neurons also explains the large increase in DOPAC levels in relation to DA values observed in early diencephalic cultures.

Apart from the sex differences found in the absence of sex steroids, region-specific modulation of DA levels by sex steroids occurred. Both estradiol and testosterone reduced DA in diencephalic but not in mesencephalic cultures, irrespective of the gender. Downregulation of DA by estrogen and androgen has also been observed in the hypothalamus of the adult male and female rat in vivo (Engel et al., 1979; Dupont et al., 1981; Battaner et al., 1987) and seems to be due to an inhibition of TH activity (Luine et al., 1977). Whether gonadal steroids affect DA content, DA turnover, and/or TH activity in mesencephalic dopaminergic neurons in vivo is controversial (Luine et al., 1977; Engel et al., 1979; Alderson and Baum, 1981; Dupont et al., 1981; Lookingland and Moore, 1984; Abreu et al., 1988).

The results of the present study support conclusions based on previous observations on sex differences in "specific" uptake of exogenous radiolabeled DA displayed by dopaminergic neurons in vitro (Engele et al., 1989b; Reisert et al., 1989). Transmitter uptake and content can be regarded as valid parameters of neu- ronal activity. If we take the present and the previous data logether, it follows that a diencephalic neuron is characterized by a higher specific uptake of exogenous DA as well as a higher content of endogenous DA in females than in males, whereas a mesencephalic neuron shows a higher specific uptake in males but no sex differences with respect to DA content. It appears that sex-related developmental processes act on different parameters of dopaminergic activity independently and in a region-specific fashion. Sex differences in dopaminergic activity have previously been observed in the hypothalamus of the adult rat in vivo (Moore, 1987) and, transiently, during early postnatal development of the rat midbrain (Stanley and Watts, 1985). The sex differences in cell numbers and/or neuronal activity described here may not be very large, and extrapolation to in vivo conditions may be limited. On the other hand, it is possible that sex differences in the early prenatal period have escaped detection because of the complex situation of the developing brain in vivo. If male and female dopaminergic neurons differ in numbers or activity in vivo before or at the onset of the now-recognized critical period, they could be a significant factor in the induction of a sexually dimorphic circuitry. 


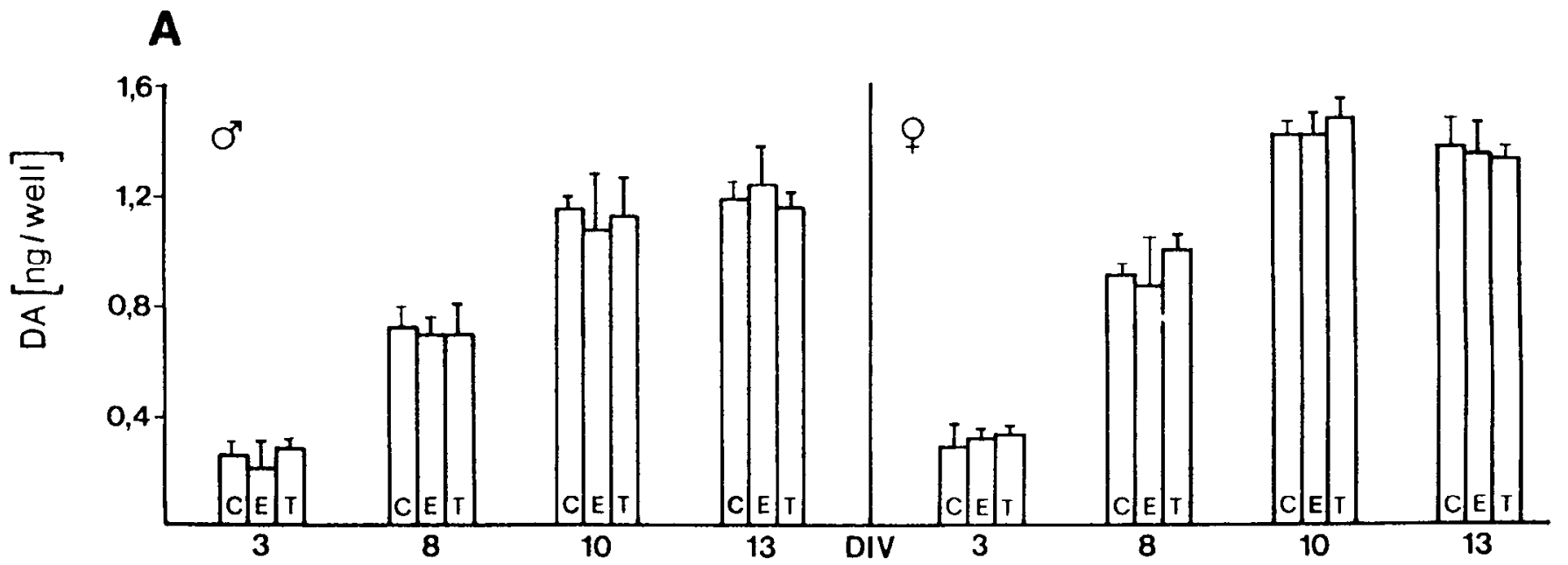

B

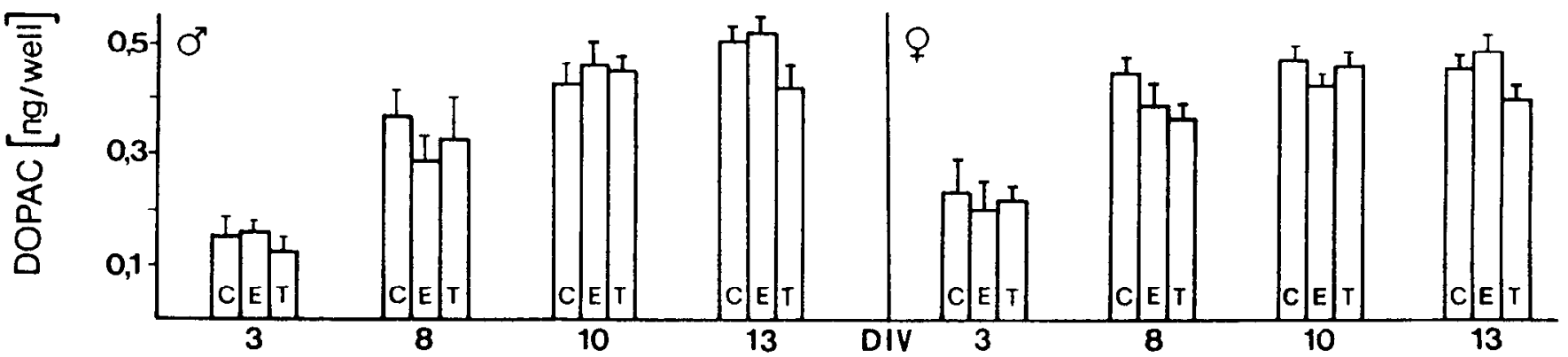

Figure 6. Effects of chronic administration of testosterone $(T)$ and $17 \beta$-estradiol $(E)$ on DA $(A)$ and DOPAC $(B)$ contents of mesencephalic cultures. Bars represent means \pm SD from four experiments.

\section{References}

Abreu P, Hernandez G, Calzadilla CH, Alonso R (1988) Reproductive hormones control striatal tyrosine hydroxylase activity in the male rat. Neurosci Lett 95:213-217.

Alderson LM, Baum MJ (1981) Differential effects of gonadal steroids on dopamine metabolism in mesolimbic and nigro-striatal pathways of male rat brain. Brain Res 218:189-206.

Amold AP, Gorski RA (1984) Gonadal steroid induction of structural sex differences in the central nervous system. Annu Rev Neurosci 7: 413-442.

Battaner E, Del Castillo AR, Guerra M, Mas M (1987) Gonadal influences on spinal cord and brain monoamines in male rats. Brain Res 425:391-394.

Berthois Y, Katzenellenbogen JA, Katzenellenbogen BS (1986) Phenol red in tissue culture media is a weak estrogen: implications concerning the study of estrogen-responsive cells in culture. Proc Natl Acad Sci USA 83:2496-2500.

Beyer C, Epp B, Fassberg J, Reisert I, Pilgrim C (1990) Region- and sex-related differences in maturation of astrocytes in dissociated cell cultures of embryonic rat brain. Glia 3:55-64.

Demarest KT, McKay DW, Riegle GD, Moore KE (1981) Sexual differences in tuberoinfundibular dopamine nerve activity induced by neonatal androgen exposure. Neuroendocrinology 32:108-113.

Dupont A, di Paolo T, Gagne B, Barden N (1981) Effects of chronic estrogen treatment on dopamine concentrations and turnover in discrete brain nuclei of ovariectomized rats. Neurosci Lett 22:69-74.

Engel J, Ahlenius S, Almgren O, Carlsson A (1979) Effects of gonadectomy and hormone replacement on brain monoamine synthesis in male rats. Pharmacol Biochem Behav 10:149-154.
Engele J, Pilgrim C, Kirsch M, Reisert I (1989a) Different developmental potentials of diencephalic and mesencephalic dopaminergic neurons in vitro. Brain Res 483:98-109.

Engele J, Pilgrim C, Reisert I (1989b) Sexual differentiation of mesencephalic dopaminergic neurons in vitro: effects of sex and gonadal hormones. Int J Dev Neurosci 7:603-611.

Feldman SC, Bloch E (1978) Developmental pattern of testosterone synthesis by fetal rat testes in response to luteinizing hormone. Endocrinology 102:999-1007.

Fung YK, Lau Y-S (1989) Effects of prenatal nicotine exposure on rat striatal dopaminergic and nicotinic systems. Pharmacol Biochem Behav 33:1-6.

Gorski RA, Jacobson CD (1982) Sexual differentiation of the brain. Front Horm Res 10:1-14.

Graefe K-H, Bönisch H (1989) The transport of amines across the axonal membranes of noradrenergic and dopaminergic neurones. In: Handbook of experimental pharmacology (catecholamines II) (Weiner N, Trendelenburg U, eds), pp 193-245. New York: Springer.

Leret ML, González MI, Tranque P, Fraile A (1987) Influence of sexual differentiation on striatal and limbic catecholamines. Comp Biochem Physiol 86C:299-303.

Lichtensteiger W, Schlumpf M (1984) Prenatal neuropharmacology: implications for neuroendocrine development. In: Fetal neuroendocrinology (Ellendorff F, Gluckman PD, Parvizi N, eds), pp 59-70. Ithaca, NY: Perinatology.

Lichtensteiger W, Ribary U, Schlumpf M, Odermatt B, Widmer HR (1988) Prenatal adverse effects of nicotine on the developing brain. In: Progress in brain research, Vol 73 (Boer GJ, Feenstra MGP, Mirmiran M, Swaab DF, Van Haaren F, eds), pp 137-157. Amsterdam: Elsevier. 
Lookingland KJ, Moore KE (1984) Effects of estradiol and prolactin on incertohypothalamic dopaminergic neurons in the male rat. Brain Res 323:83-91.

Luine VN, McEwen BS, Black IB (1977) Effect of $17 \beta$-estradiol on hypothalamic tyrosine hydroxylase activity. Brain Res 120:188-192.

MacLusky NJ, Naftolin F (1981) Sexual differentiation of the central nervous system. Science 211:1294-1303.

McEwen BS (1981) Neural gonadal steroid actions. Science 211:13031311.

Moore KE (1987) Hypothalamic dopaminergic neuronal systems. In: Psychopharmacology. The third generation of progress (Meltzer HY, ed), pp 127-139. New York: Raven.

Pasqualini JR, Kincl FA (1985) Hormones and the fetus, Vol 1. Frankfurt: Pergamon.

Picon R (1976) Testosterone secretion by foetal rat testes in vitro. J Endocrinol 71:231-238.

Reisert I, Engele J, Pilgrim C (1989) Early sexual differentiation of diencephalic dopaminergic neurons of the rat in vitro. Cell Tissue Res 255:411-417.

Reisert I, Schuster R, Zienecker R, Pilgrim C (1990) Prenatal development of mesencephalic and diencephalic dopaminergic systems in the male and female rat. Dev Brain Res 53:222-229.

Ribary U, Schlumpf M, Lichtensteiger W (1986) Analysis by HPLCEC of metabolites of monoamines in fetal and postnatal rat brain. Neuropharmacology 25:981-986.

Simerly RB, Swanson LW, Gorski RA (1985a) The distribution of monoaminergic cells and fibers in a periventricular preoptic nucleus involved in the control of gonadotropin release: immunocytochemical evidence for a dopaminergic sexual dimorphism. Brain Res 330:5564.

Simerly RB, Swanson LW, Handa RJ, Gorski RA (1985b) Influence of perinatal androgen on the sexually dimorphic distribution of tyrosine hydroxylase-immunoreactive cells and fibers in the anteroventral periventricular nucleus of the rat. Neuroendocrinology 40: 501-51r.

Smith PK, Krohn RI, Hermanson GT, Mallia AK, Gartner FH, Provenzano MD, Fujimoto EK, Goeke NM, Olson BJ, Klenk DC (1985) Measurement of protein using bicinchoninic acid. Anal Biochem 150: $76-85$.

Stanley HF, Watts AG (1985) Changes in catecholamine concentrations in the hypothalamus and region of the midbrain raphe in male and female rats during postnatal development. Neurosci Lett 62:7580.

Vaccari A (1980) Sexual differentiation of monoamine neurotransmitters. In: Biogenic amines in development (Parvez $\mathrm{H}$, Parvez $\mathrm{S}$, eds), pp 327-352. Amsterdam: Elsevier/North-Holland.

Weisz J, Ward IL (1980) Plasma testosterone and progesterone titers of pregnant rats, their male and female fetuses, and neonatal offspring. Endocrinology 106:306-316.

Wilson WE, Agrawal AK (1979) Brain regional levels of neurotransmitter amines as neurochemical correlates of sex-specific ontogenesis in the rat. Dev Neurosci 2:195-200. 